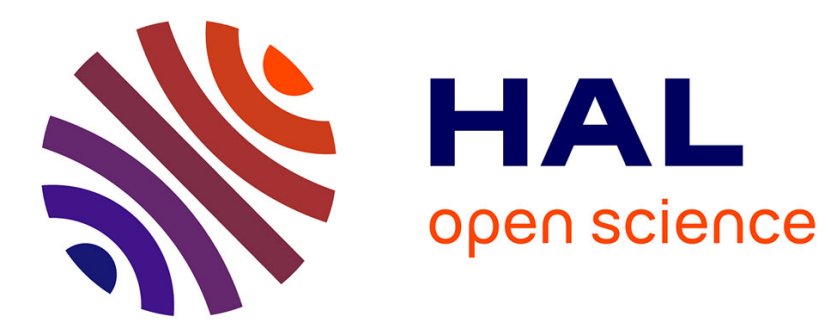

\title{
Linear impulse response in hot round jets
}

\author{
Lutz Lesshafft, Patrick Huerre
}

\section{To cite this version:}

Lutz Lesshafft, Patrick Huerre. Linear impulse response in hot round jets. Physics of Fluids, 2007, 19 (2), pp.024102. 10.1063/1.2437238 . hal-01023086

\section{HAL Id: hal-01023086 \\ https://hal-polytechnique.archives-ouvertes.fr/hal-01023086}

Submitted on $20 \mathrm{Jul} 2014$

HAL is a multi-disciplinary open access archive for the deposit and dissemination of scientific research documents, whether they are published or not. The documents may come from teaching and research institutions in France or abroad, or from public or private research centers.
L'archive ouverte pluridisciplinaire HAL, est destinée au dépôt et à la diffusion de documents scientifiques de niveau recherche, publiés ou non, émanant des établissements d'enseignement et de recherche français ou étrangers, des laboratoires publics ou privés. 


\section{AIP | Physics of Fluids}

\section{Linear impulse response in hot round jets}

Lutz Lesshafft and Patrick Huerre

Citation: Physics of Fluids (1994-present) 19, 024102 (2007); doi: 10.1063/1.2437238

View online: http://dx.doi.org/10.1063/1.2437238

View Table of Contents: http://scitation.aip.org/content/aip/journal/pof2/19/2?ver=pdfcov

Published by the AIP Publishing

Articles you may be interested in

Linear stability and acoustic characteristics of compressible, viscous, subsonic coaxial jet flow Phys. Fluids 25, 084102 (2013); 10.1063/1.4816368

The impulse response of a high-speed jet forced with localized arc filament plasma actuators Phys. Fluids 24, 125104 (2012); 10.1063/1.4772191

Linear stability of a compressible coaxial jet with continuous velocity and temperature profiles Phys. Fluids 20, 074102 (2008); 10.1063/1.2946443

Frequency selection in globally unstable round jets

Phys. Fluids 19, 054108 (2007); 10.1063/1.2732247

Energy growth of initial perturbations in two-dimensional gravitational jets

Phys. Fluids 14, 289 (2002); 10.1063/1.1425411

\section{A|P| Journal of}

Journal of Applied Physics is pleased to announce André Anders as its new Editor-in-Chief 


\title{
Linear impulse response in hot round jets
}

\author{
Lutz Lesshafft ${ }^{\mathrm{a})}$ and Patrick Huerre \\ Laboratoire d'Hydrodynamique (LadHyX), CNRS - École Polytechnique, 91128 Palaiseau, France
}

(Received 11 May 2006; accepted 16 November 2006; published online 27 February 2007)

\begin{abstract}
The linear impulse response of axisymmetric jets is examined for a family of variable-temperature profiles typical of the potential core. The influence of jet heating, shear layer thickness, and Reynolds and Mach numbers on the spatiotemporal stability of both axisymmetric and helical modes is investigated. The linear impulse response is retrieved from a numerical solution of the spatial eigenvalue problem, which is derived from the fully compressible equations of motion. Changes in the spatiotemporal stability of heated versus isothermal jets are shown to arise solely from the effect of the baroclinic torque. By considering the full linear impulse response, the competition between jet column modes and shear layer modes is characterized. Jet column modes are only found to occur for axisymmetric disturbances. In thin shear layer jets, the jet column mode is shown to prevail at low group velocities, whereas axisymmetric and helical shear layer modes dominate at high group velocities. The absolute mode of zero group velocity is found to always be of the jet column type. Although only convectively unstable, the maximum growth rates of the shear layer modes greatly exceed those of the jet column modes in thin shear layer jets. In thick shear layer jets, axisymmetric modes of mixed jet column/shear layer type arise. The weakened maximum growth rate of mixed modes accounts for the dominance of helical modes in temporal stability studies of thick shear layer jets. (C) 2007 American Institute of Physics. [DOI: 10.1063/1.2437238]
\end{abstract}

\section{INTRODUCTION}

The theoretical and experimental studies of Monkewitz and Sohn ${ }^{1}$ and Monkewitz et al. ${ }^{2}$ have provided strong evidence that the occurrence of self-sustained oscillations in sufficiently heated jets is connected to a transition from convective to absolute instability of the unperturbed flow state. The objective of the present investigation is to fully characterize the linear instability modes that are observed in hot jets as a function of their group velocity. Such instability modes precisely constitute the ingredients of the linear impulse response. A family of parallel velocity and temperature profiles typical of the potential core region in spatially developing jets is considered, and their spatiotemporal stability characteristics are determined from the full linear impulse response wave packet. An analysis of the dispersion relation allows to identify the physical mechanism by which hot jets become absolutely unstable. It should be understood that all results obtained for hot jets equally pertain to cases where density variations are due to the mixing of nonhomogeneous fluids as, for instance, helium jets in air. ${ }^{3-5}$

The effect of temperature variations on the spatial instability of axisymmetric jets has been studied theoretically by Michalke. ${ }^{6,7}$ In agreement with earlier predictions drawn from the analysis of plane shear layers (Blumen et $\mathrm{ll}^{8}$ ), heating of the jet with respect to the surrounding fluid was shown to promote the spatial growth of externally forced perturbations. Michalke identified a "regular" and an "irregular" unstable axisymmetric mode. Unexplained at the time, the eigenvalues of these two modes seemed to interchange as the ambient-to-jet temperature ratio fell below 0.7. Once the

\footnotetext{
${ }^{a)}$ Electronic mail: lutz.lesshafft@ladhyx.polytechnique.fr
}

concepts of absolute and convective instability ${ }^{9,10}$ had been introduced to fluid mechanics, Huerre and Monkewitz ${ }^{11}$ later interpreted the "irregular" mode as an upstream-traveling $k^{-}$ branch, and the apparent mode interchange as a result of the onset of absolute instability.

The occurrence of absolute instability in hot round jets without counterflow has been firmly established by Monkewitz and Sohn. ${ }^{1}$ These authors investigated the transition from convective to absolute instability in terms of the temperature ratio, the Mach number, and the shear layer thickness relative to the jet radius. Absolute instability was found to first set in for axisymmetric perturbations, at a critical temperature ratio of 0.72 and finite shear layer thickness. In contrast, Pavithran and Redekopp ${ }^{12}$ demonstrated that nonhomogeneous plane shear layers only display absolute instability in the presence of counterflow.

Jendoubi and Strykowski ${ }^{13}$ extended the analysis of Monkewitz and Sohn ${ }^{1}$ to jets with ambient coflow and counterflow. Their study remains the most comprehensive spatiotemporal analysis of axisymmetric jets to this day. Restricted to axisymmetric disturbances, their investigation revealed the presence of two distinct instability modes. In a thin shear layer jet, the first of these axisymmetric modes was shown to be closely related to the plane shear layer instability described by Pavithran and Redekopp: ${ }^{12}$ All perturbations are concentrated within the jet shear layer region, and absolute instability only occurs in the presence of sufficiently strong counterflow. This mode will be denoted as the shear layer mode throughout this paper. The second mode was shown to be identical with the absolute instability mode discovered by Monkewitz and Sohn. ${ }^{1}$ Its pressure eigenfunction peaks on the jet axis. Henceforth, this mode will be denoted as the jet column mode. 
While the study of Jendoubi and Strykowski ${ }^{13}$ clearly identifies the convective/absolute transition of the shear layer and jet column modes as a function of temperature ratio and external flow, the respective roles of these two competing modes in a given base flow cannot be understood by considering the absolute instability mode of zero group velocity alone. Arbitrary perturbations in real flows will always create nonzero group velocity modes that may experience strong temporal amplification. The aim of the present paper is to provide a spatiotemporal instability analysis in terms of the full linear impulse response. The whole wave packet evolving from an initial Dirac-type perturbation according to the linear equations of motion is considered. This wave packet is composed of a continuous spectrum of jet column and shear layer modes, each one traveling in the axial direction at a distinct group velocity $v_{g}$. The simultaneous growth of jet column and shear layer modes can therefore be characterized as a function of their group velocity. For a review of spatiotemporal instability theory, the reader is referred to Huerre. ${ }^{14}$ Unlike Ref. 13, axisymmetric as well as helical modes are considered. The inviscid results of Monkewitz and Sohn, ${ }^{1}$ and Jendoubi and Strykowski, ${ }^{13}$ are further complemented by parameter studies of the convective/absolute instability boundary at finite Reynolds and Mach numbers and for thin and thick shear layers. The analysis is restricted to situations with zero external flow without loss of generality, as the effect of coflow or counterflow on the linear impulse response wave packet in parallel jet profiles can be obtained by a simple transformation provided in Sec. II.

The paper is organized as follows: The formulation of the base flow and the mathematical model for the linear instability analysis are defined in Sec. II. The numerical solution of the dispersion relation is briefly outlined in Sec. III. The eigenvalue problem representing the compressible viscous dispersion relation is documented in the Appendix, together with further details of its numerical discretization. The linear impulse response of an isothermal thin shear layer jet is examined in Sec. IV A and compared to corresponding results obtained for a hot jet in Sec. IV B. Modifications of the dispersion relation lead to identify the physical mechanism responsible for the occurrence of absolute instability in hot jets. The linear impulse response of a thick shear layer jet as well as the influence of the Reynolds and Mach numbers on the onset of absolute instability are examined in Sec. IV C. The paper concludes with a summary of the main results.

\section{PROBLEM FORMULATION}

The linear impulse response is determined for an axisymmetric compressible jet base flow of density $\rho_{b}$, temperature $T_{b}$, pressure $p_{b}$, and axial velocity $u_{b}$. The base flow is considered to be parallel in the axial direction and swirl free; the radial and azimuthal velocity components $v_{b}$ and $w_{b}$ therefore are zero. The flow geometry is formulated in cylindrical coordinates $(x, r, \phi)$. All flow variables are given in nondimensional form, scaled with respect to the jet radius $R$ and the jet centerline values $U_{c}, \rho_{c}$, and $T_{c}$. An analytical expression for base flow velocity profiles, typical of the potential core region in laboratory jets, is taken from Michalke, ${ }^{6}$

$$
u_{b}(r)=\frac{1}{2}+\frac{1}{2} \tanh \left[\frac{R}{4 \theta}\left(\frac{1}{r}-r\right)\right] .
$$

The velocity profile is characterized by the parameter $R / \theta$, where $\theta$ denotes the momentum thickness of the shear layer. The radial temperature variation for a given ambient-to-jet temperature ratio $S=T_{\infty} / T_{C}$ is linked to the velocity profile via the Crocco-Busemann relation, ${ }^{7}$

$$
T_{b}(r)=S+(1-S) u_{b}(r)+\frac{\gamma-1}{2} \operatorname{Ma}^{2}\left[1-u_{b}(r)\right] u_{b}(r) .
$$

The Mach number is defined as $\mathrm{Ma}=U_{c} / c_{c}$, with $c_{c}$ the speed of sound on the jet centerline, and the ratio of specific heats $\gamma$ is chosen as 1.4 throughout this study. The pressure $p_{b}$ in the unperturbed jet is constant and can be obtained from the equation of state for a perfect gas,

$$
p=\frac{1}{\gamma \mathrm{Ma}^{2}} \rho T .
$$

On the centerline, where $\rho_{b}$ and $T_{b}$ are unity, one finds

$$
p_{b}=\frac{1}{\gamma \mathrm{Ma}^{2}} \text {. }
$$

The density profile is then given as

$$
\rho_{b}(r)=T_{b}(r)^{-1} .
$$

The flow is assumed to be governed by the compressible equations of continuity, momentum, and energy, written in total flow quantities as

$\frac{d \rho}{d t}=-\rho \operatorname{div} \mathbf{u}$,

$\rho \frac{d \mathbf{u}}{d t}=-\operatorname{grad} p+\operatorname{div} \boldsymbol{\tau}$,

$\rho \frac{d}{d t}\left(\frac{p}{\rho}\right)=-(\gamma-1) p \operatorname{div} \mathbf{u}+(\gamma-1) \boldsymbol{\tau}: \boldsymbol{\varepsilon}+\frac{\gamma}{\operatorname{Re}(\operatorname{Pr})} \Delta\left(\frac{p}{\rho}\right)$,

with the Reynolds and Prandtl numbers defined as

$$
\operatorname{Re}=\frac{\rho_{c} R U_{c}}{\mu}, \quad \operatorname{Pr}=c_{p} \frac{\mu}{\kappa} .
$$

The viscous stress tensor $\boldsymbol{\tau}$ and the rate of strain tensor $\boldsymbol{\varepsilon}$ are given by

$$
\boldsymbol{\tau}=-\frac{2}{3 \operatorname{Re}}(\operatorname{div} \mathbf{u}) \mathbf{I}+\frac{2}{\operatorname{Re}} \boldsymbol{\varepsilon},
$$




$$
\boldsymbol{\varepsilon}=\frac{1}{2}\left(\operatorname{grad} \mathbf{u}+\operatorname{grad}^{T} \mathbf{u}\right)
$$

The dynamical viscosity $\mu$ and thermal conductivity $\kappa$ are taken to be constant throughout the flow and related by a Prandtl number of unity.

Small perturbations $\left(\rho^{\prime}, u^{\prime}, v^{\prime}, w^{\prime}, p^{\prime}\right)$ to the base flow, where $\left(u^{\prime}, v^{\prime}, w^{\prime}\right)$ denote the $(x, r, \phi)$ components of the perturbation velocity, are now expressed as normal modes of complex axial wave number $k$, integer azimuthal wave number $m$, and complex angular frequency $\omega$ according to

$$
\left[\begin{array}{c}
\rho^{\prime} \\
u^{\prime} \\
v^{\prime} \\
w^{\prime} \\
p^{\prime}
\end{array}\right](x, r, \phi, t)=\left[\begin{array}{c}
D(r) \\
H(r) \\
i F(r) \\
G(r) \\
P(r)
\end{array}\right] e^{i(k x+m \phi-\omega t)}+\mathrm{c} . \mathrm{c} .
$$

The notation $(H, F, G, P)$ in Eq. (12) has been chosen to correspond to the incompressible problem formulation of Khorrami et al. ${ }^{15}$ Substitution of (12) into the equations of motion (6)-(8), linearized about the base flow, yields a linear system of ordinary differential equations. In the same manner as in Ref. 15, this system is cast in the form of a generalized eigenvalue problem which, for prescribed values of the frequency $\omega$, admits spatial eigenvalues $k$ and corresponding complex eigenfunctions $(D, H, F, G, P)$. The compressible spatial eigenvalue problem is stated explicitly in the Appendix.

At large times $t$, the linear impulse response along each spatiotemporal ray $x / t=$ const is dominated by the absolute instability mode in the reference frame moving at $v=x / t$ with respect to the laboratory frame (see Ref. 16). In order to construct numerically the linear impulse response, values of $\omega\left(v_{g}\right), k\left(v_{g}\right)$ for a given group velocity $v_{g}$ can therefore be computed as the absolute instability modes in the comoving reference frame $(\tilde{r}, \tilde{x})=\left(r, x-v_{g} t\right)$, where the axial base flow velocity profile becomes $\tilde{u}_{b}(r)=u_{b}(r)-v_{g}$. The resulting values $\widetilde{\omega}_{0}, \tilde{k}_{0}$ are then transformed back into the laboratory reference frame according to the relations $\omega\left(v_{g}\right)=\widetilde{\omega}_{0}+\widetilde{k}_{0} v_{g}$ and $k\left(v_{g}\right)=\tilde{k}_{0}$. For each azimuthal wave number $m$, results are presented in the laboratory frame in terms of real frequency $\omega_{r}\left(v_{g}\right)$ and spatiotemporal growth rate $\sigma\left(v_{g}\right)$ $=\omega_{i}\left(v_{g}\right)-k_{i}\left(v_{g}\right) v_{g}=\tilde{\omega}_{0, i}$ along each ray $x / t=v_{g}$.

By construction, it is clear that the effect of external coflow or counterflow on the linear impulse response merely results in an offset of $v_{g}$ and a Doppler shift of the real frequency. ${ }^{17}$ From the distributions $\omega_{r}\left(v_{g}\right), \sigma\left(v_{g}\right), k_{r}\left(v_{g}\right)$, $k_{i}\left(v_{g}\right)$ in a situation with zero external flow, the corresponding distributions $\tilde{\omega}_{r}\left(v_{g}\right), \widetilde{\sigma}\left(v_{g}\right), \tilde{k}_{r}\left(v_{g}\right), \tilde{k}_{i}\left(v_{g}\right)$ in a situation with external flow $\tilde{u}_{b}=u_{b}+u_{e}$ are obtained as

$$
\begin{aligned}
& \tilde{\omega}_{r}\left(v_{g}\right)=\omega_{r}\left(v_{g}-u_{e}\right)-u_{e} k_{r}\left(v_{g}-u_{e}\right), \\
& \widetilde{\sigma}\left(v_{g}\right)=\sigma\left(v_{g}-u_{e}\right), \\
& \tilde{k}_{r}\left(v_{g}\right)=k_{r}\left(v_{g}-u_{e}\right), \\
& \tilde{k}_{i}\left(v_{g}\right)=k_{i}\left(v_{g}-u_{e}\right) .
\end{aligned}
$$

\section{NUMERICAL METHOD}

In order to compute values of the absolute frequency and wave number, Monkewitz and Sohn, ${ }^{1}$ as well as Jendoubi and Strykowski, ${ }^{13}$ used a shooting method to numerically solve the dispersion relation in the form of a single-variable ordinary differential equation. ${ }^{6}$ The numerical procedure used in the present study closely follows the Chebyshev collocation method described by Ash and Khorrami, ${ }^{18}$ which only had to be extended to include compressible effects. For a given set of parameters $\left(m, v_{g}, R / \theta, S, \mathrm{Re}, \mathrm{Ma}\right)$, the eigenvalue problem (A1)-(A5) is discretized and solved numerically for the spatial branches $k(\omega)$. A code provided by Olendraru and Sellier ${ }^{19}$ has been adapted to the compressible jet problem. The complex pair $\left(k_{0}, \omega_{0}\right)$ is then determined by tracking the point where a $k^{+}$and a $k^{-}$branch pinch in the complex $k$ plane. ${ }^{8,9}$ For this purpose, the iterative search algorithm described in Ref. 1 was found to be reliable and very time efficient. The transformation used for an appropriate distribution of collocation points for thin shear layer jet profiles is given in the Appendix. A validation of the numerical procedure has been presented in Ref. 20 by comparing the $\sigma\left(v_{g}\right)$ distribution computed from the dispersion relation for $m=0$ to the results of a direct numerical simulation of the axisymmetric linear Navier-Stokes equations.

\section{RESULTS}

\section{A. Incompressible inviscid jet}

We first consider the linear impulse response of an isothermal jet $(S=1)$ in the inviscid, zero-Mach-number limit. The velocity profile parameter for this thin shear layer example is chosen as $R / \theta=20$. While such a profile may not be considered "thin" by some readers, comparison with the results discussed in Sec. IV C will show that the separation of scales between jet radius and shear layer thickness is sufficient to allow a discussion of the spatiotemporal characteristics of arbitrarily thin shear layer jets. The spatiotemporal growth rate $\sigma$, real frequency $\omega_{r}$, real wave number $k_{r}$, and spatial growth rate $-k_{i}$ of the axisymmetric $(m=0)$ component are presented in Fig. 1 (thin lines) as functions of their group velocity $v_{g}$.

A discontinuity in the spectrum at $v_{g}=0.182$ divides the wave packet into two regions, each composed of a distinct class of instability modes. The low group velocity modes correspond to absolute instability modes in jets with zero or moderate counterflow. According to Jendoubi and Strykowki, ${ }^{13}$ these modes are of the jet column type. Modes traveling at group velocities $v_{g}>0.182$ correspond to absolute instability modes in jets with strong counterflow, char- 

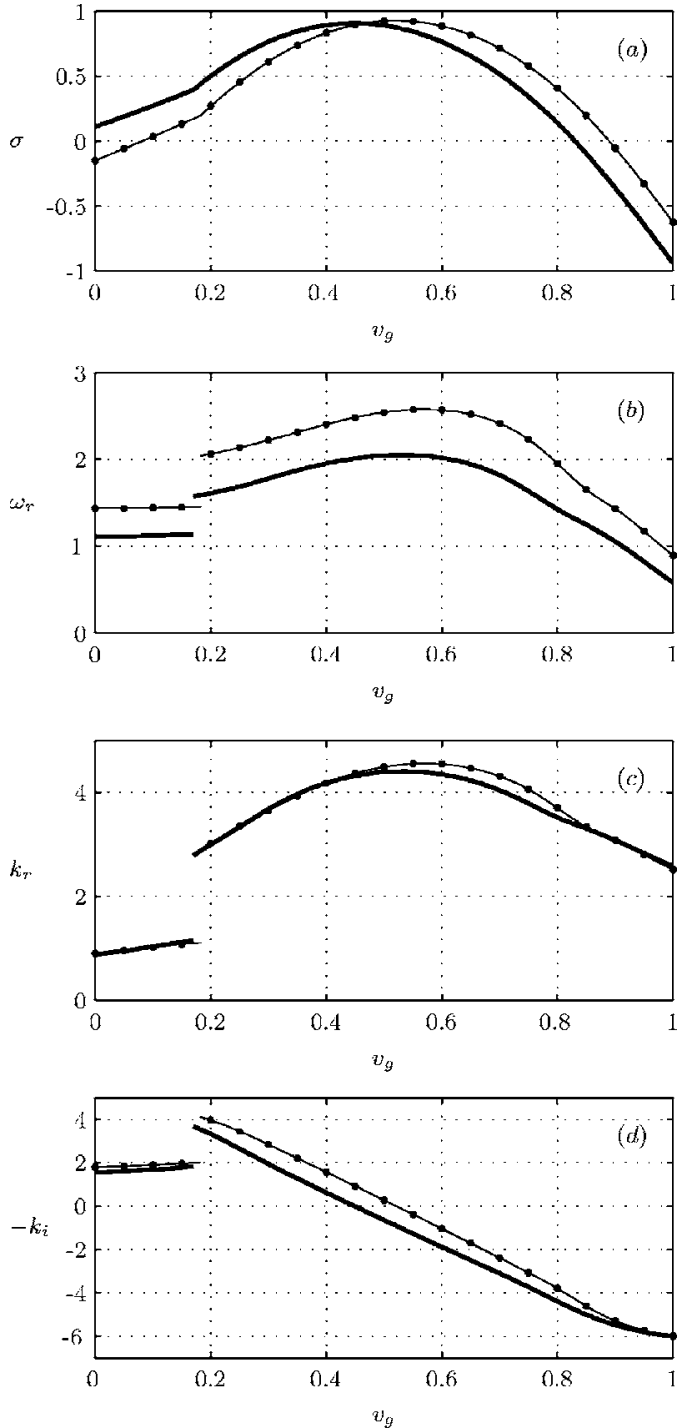

FIG. 1. Axisymmetric linear impulse response for flow parameters $R / \theta$ $=20, \operatorname{Re}=\infty, \mathrm{Ma}=0$. Isothermal case $S=1$ (thin line); heated case $S=0.5$ (thick line); heated case $S=0.5$ in the absence of baroclinic torque $(\bullet$ ). (a) Spatiotemporal growth rate; (b) real frequency; (c) real wave number; (d) spatial growth rate, all as functions of group velocity $v_{g}$.

acterized as being of the shear layer type. ${ }^{13}$ This distinct jet column/shear layer character is confirmed in Fig. 2: For two profile parameters $R / \theta=20$ (thin line, same as in Fig. 1) and $R / \theta=40$ (thick line), the $\sigma\left(v_{g}\right)$ distributions are compared when scaled with respect to the jet radius $R$ [Fig. 2(a)] and the shear layer momentum thickness $\theta$ [Fig. 2(b)], respectively. The growth rate is found to scale with $R$ for the low group velocity modes and with $\theta$ for the high group velocity modes.

Jendoubi and Strykowki ${ }^{13}$ have shown that the absolute instability mode in jets with variable external flow arises from pinching events involving the same unstable $k^{+}$branch, but two distinct $k^{-}$branches for the shear layer and jet column modes. It should be pointed out that only one of these two pinching events, i.e., the one occurring at a higher value of $\sigma$, is to be regarded as physically relevant. ${ }^{21}$ The interaction of a unique $k^{+}$branch with one out of several $k^{-}$ branches has also been reported by Loiseleux et al. $^{22}$ to pro-
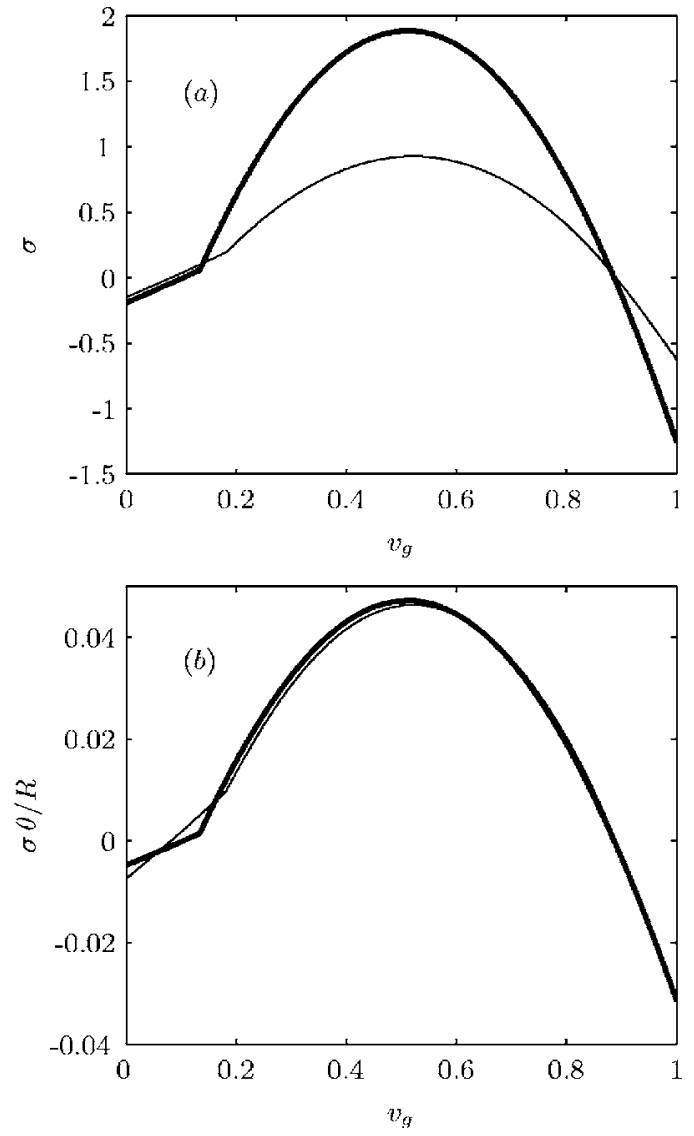

FIG. 2. Comparison of the spatiotemporal growth rates $\sigma$ in jets for $R / \theta$ $=20$ (thin line) and $R / \theta=40$ (thick line); $m=0, S=1, \operatorname{Re}=\infty, \mathrm{Ma}=0$. (a) $\sigma$ scaled with jet radius $R$; (b) $\sigma$ scaled with shear layer thickness $\theta$.

duce distinct absolute instability modes in swirling jets with counterflow. The pinching between branches in the complex $k$ plane is presented in Fig. 3 for the case considered here, for two group velocities $v_{g}=0$ and $v_{g}=0.3$. The displayed branches are obtained as solutions of the dispersion relation for given values $\omega=\omega_{r}+i \omega_{i}$, where $\omega_{r}$ is continuously varied for three fixed values of $\omega_{i}$. Consistent with the notation of Ref. 22, let $k_{1}^{-}$denote the spatial branch in our problem which, upon pinching with the $k^{+}$branch gives rise to a jet column mode, and $k_{2}^{-}$its counterpart for the shear layer mode. At $v_{g}=0$ [Fig. 3(a)], the $k^{+}, k_{1}^{-}$, and $k_{2}^{-}$branches are well separated for $\omega_{i}=0$ (thin solid lines). With $\omega_{i}=\omega_{0, i}$ $=-0.150$ (thick lines), the $k^{+}$and $k_{1}^{-}$branches pinch at the saddle point $k_{0}=0.901-1.808 i$ for a real frequency $\omega_{0, r}$ $=1.436$. These values correspond to those plotted in Fig. 1 at $v_{g}=0$. If $\omega_{i}$ is lowered further, a second saddle point is eventually formed by the merged $k^{+} / k_{1}^{-}$branch and the $k_{2}^{-}$branch (dashed lines). However, formal solutions of the dispersion relation for $\omega_{i}<\omega_{0, i}$ are noncausal, ${ }^{14}$ and therefore do not correspond to physical situations. Only the pinching events between $k^{+}$and $k^{-}$branches occurring at the highest value of $\omega_{i}$ are taken into account in this study.

Corresponding $k$-branch diagrams in Fig. 3(b) display that the relevant saddle point for $v_{g}=0.3$ is formed between the $k^{+}$and $k_{2}^{-}$branches; the associated instability mode is of the shear layer type, as for all group velocities $v_{g}>0.182$. A 
(a)

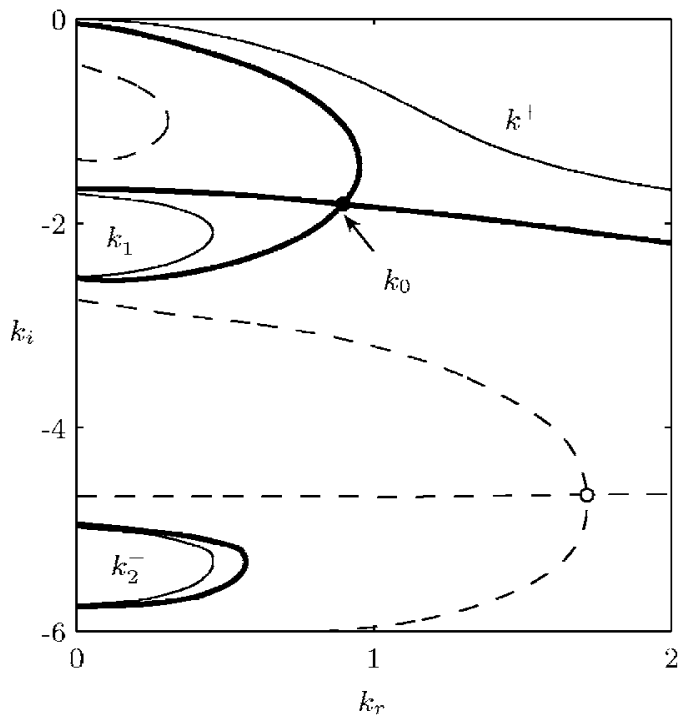

(b)

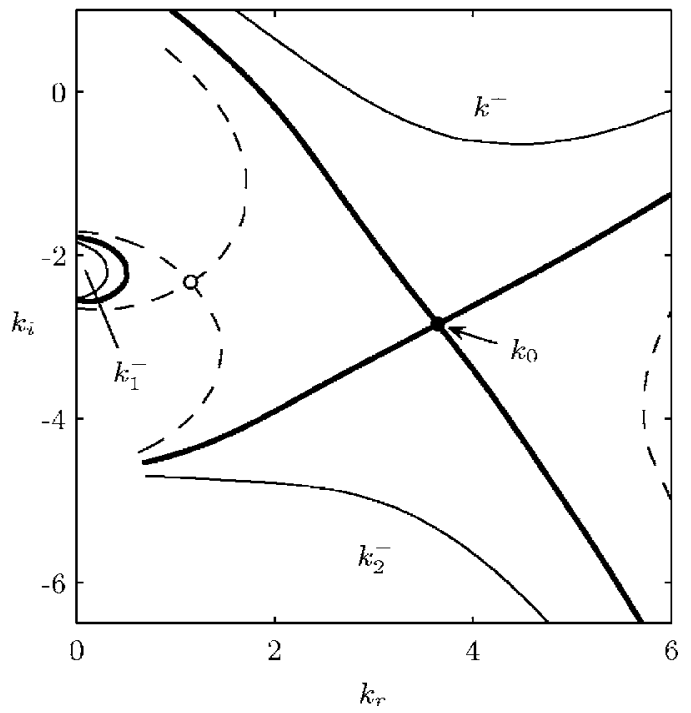

FIG. 3. Branches in the complex $k$ plane for $R / \theta=20, S=1, \operatorname{Re}=\infty$, and $\mathrm{Ma}=0$. (a) $v_{g}=0$, three constant values of $\omega_{i}: 0$ (thin solid), 0.15 (thick solid), 0.63 (dashed); (b) $v_{g}=0.3$, three constant values of $\omega_{i}: 0.8$ (thin solid), 0.61 (thick solid), 0.45 (dashed).

different scenario is observed in thick shear layer jets, as discussed in Sec. IV C.

According to Fig. 1, a real wave number $k \sim 1$ is found to be typical of the jet column modes. This value corresponds to a wavelength $\lambda \sim 2 \pi$, large when compared to the shear layer mode wavelengths, and a real phase velocity $\omega_{r} / k_{r}$ larger than the jet centerline velocity. The parabolashaped variation of $\sigma\left(v_{g}\right)$ at group velocities $v_{g}>0.182$ is typical of the Kelvin-Helmholtz instability for a plane shear layer. The mode of maximum spatiotemporal growth, which corresponds to the most unstable temporal mode with $\omega_{i, \max }=\sigma_{\max }$ and $k_{i}=0$ (see, for instance, Huerre ${ }^{14}$ ), is of the shear layer type. Since $\sigma(0)<0$ and $\sigma_{\max }>0$, the isothermal jet is convectively unstable, in agreement with Monkewitz and Sohn. ${ }^{1}$ (a)

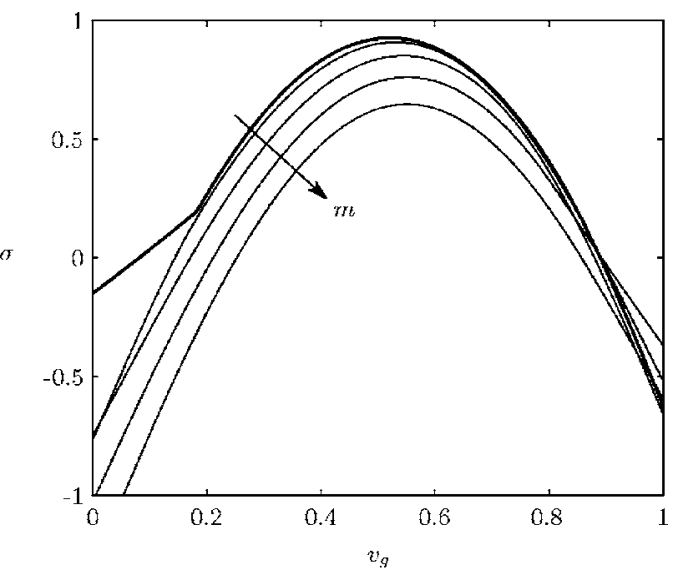

(b)

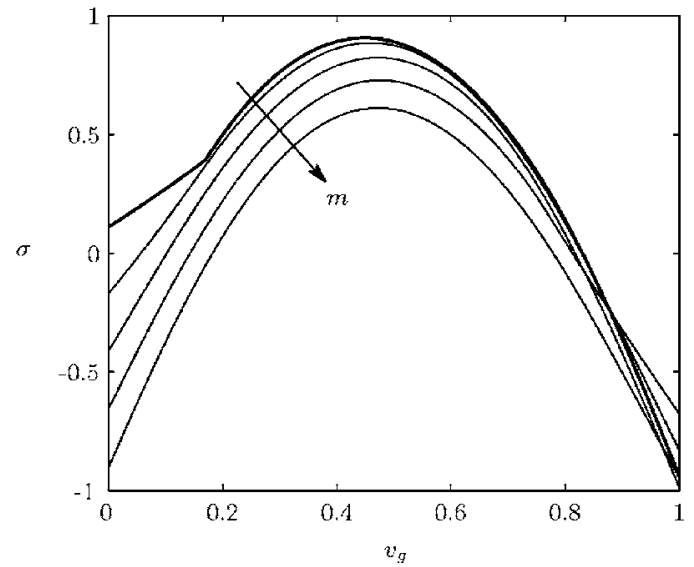

FIG. 4. Growth rates $\sigma$ of the axisymmetric $m=0$ mode (thick line) and azimuthal modes $m=1,2,3,4$ in a thin shear layer jet; $R / \theta=20, \operatorname{Re}=\infty$, $\mathrm{Ma}=0$. (a) $S=1$; (b) $S=0.5$.

The growth rates of the first four azimuthal modes $(\mathrm{m}$ $=1,2,3,4)$ are compared to the $m=0$ mode in Fig. 4 . The maximum values $\sigma_{\max }$ of each individual curve are seen to slowly diminish with increasing azimuthal wave number $m$. However, the growth rates of the $m=0$ shear layer modes and the $m=1$ modes are nearly identical. All modes $m \geq 1$ are of the shear layer type, whatever the value of $v_{g}$. At low group velocities, and in particular at $v_{g}=0$, the linear impulse response is clearly dominated by the axisymmetric jet column mode.

\section{B. Influence of the temperature ratio S: Baroclinic torque}

The effect of a nonuniform temperature profile on the spatiotemporal instability of a jet is demonstrated for a temperature ratio $S=0.5$, with all other parameters identical to the isothermal case described in the previous section.

The axisymmetric linear impulse response of the heated jet is given in Fig. 1 (thick lines) for comparison with the isothermal case. For the jet column mode $\left(v_{g}<0.170\right)$, the heating is seen to give rise to an overall increase of the growth rate $\sigma$, while the real frequency takes on lower values. In agreement with the analysis of Monkewitz and Sohn, ${ }^{1}$ the $S=0.5$ case is found to be absolutely unstable $(\sigma(0)>0)$. 
The complex wave numbers of the jet column modes are hardly affected by the temperature ratio. The parabolashaped $\sigma$ distribution of the shear layer modes is shifted towards lower group velocities as compared to the isothermal case, but the maximum growth rate $\sigma_{\max }$ remains approximately the same. The growth rates of the azimuthal modes of the heated jet, displayed in Fig. 4(b), are found to display the same trend. As in isothermal jets, the axisymmetric and first azimuthal modes are in close competition for high group velocities. All azimuthal modes are convectively unstable at $S=0.5$.

It has been suggested by Soteriou and Ghoniem ${ }^{23}$ that differences in the instability characteristics of homogeneous and nonhomogeneous shear layers may be ascribed to the action of the baroclinic torque. According to these authors, the presence of a baroclinic vorticity dipole within a rolled-up eddy may explain the lateral displacement of the eddy core into the low-density stream, as well as the bias of its convection speed towards the velocity of the high-density stream. Both of these features are in qualitative agreement with numerical observations. ${ }^{23}$

Following this idea, the role of baroclinic effects in the linear impulse response of a heated jet is quantitatively assessed by solving a modified dispersion relation, in which the baroclinic torque term is counterbalanced by appropriate forcing. Only the axisymmetric case is considered here. In the presence of source terms denoted as $S_{x}$ and $S_{r}$, the linear inviscid momentum equations become

$$
\begin{aligned}
& \frac{\partial u^{\prime}}{\partial t}=-v^{\prime} \frac{\partial u_{b}}{\partial r}-u_{b} \frac{\partial u^{\prime}}{\partial x}-\frac{1}{\rho_{b}} \frac{\partial p^{\prime}}{\partial x}+S_{x}, \\
& \frac{\partial v^{\prime}}{\partial t}=-u_{b} \frac{\partial v^{\prime}}{\partial x}-\frac{1}{\rho_{b}} \frac{\partial p^{\prime}}{\partial r}+S_{r},
\end{aligned}
$$

and the azimuthal perturbation vorticity $\Omega_{\theta}^{\prime}=\operatorname{curl} \mathbf{u}^{\prime}$ is found to evolve as

$$
\begin{aligned}
\frac{\partial \Omega_{\theta}^{\prime}}{\partial t}= & v^{\prime} \frac{\partial^{2} u_{b}}{\partial r^{2}}+u_{b} \frac{\partial^{2} u^{\prime}}{\partial x \partial r}-u_{b} \frac{\partial^{2} v^{\prime}}{\partial x^{2}}+\frac{\partial u_{b}}{\partial r}\left(\frac{\partial u^{\prime}}{\partial x}+\frac{\partial v^{\prime}}{\partial r}\right) \\
& -\frac{1}{\rho_{b}^{2}} \frac{\partial \rho_{b}}{\partial r} \frac{\partial p^{\prime}}{\partial x}+\frac{\partial S_{r}}{\partial x}-\frac{\partial S_{x}}{\partial r} .
\end{aligned}
$$

In order to eliminate the effect of the baroclinic torque $(\nabla \rho$ $\times \nabla p) / \rho^{2}$, the source terms $S_{x}$ and $S_{r}$ are selected so as to satisfy the constraint,

$$
\frac{\partial S_{r}}{\partial x}-\frac{\partial S_{x}}{\partial r}=\frac{1}{\rho_{b}^{2}} \frac{\partial \rho_{b}}{\partial r} \frac{\partial p^{\prime}}{\partial x},
$$

without introducing mass sources in the continuity equation, i.e.,

$$
\frac{1}{r} \frac{\partial}{\partial r}\left(r \rho_{b} S_{r}\right)+\frac{\partial}{\partial x}\left(\rho_{b} S_{x}\right)=0
$$

A modified dispersion relation is now constructed from the forced momentum equations (14) and the unforced continuity and energy equations, together with the forcing conditions (16) and (17). The source terms $S_{x}$ and $S_{r}$ are consid-

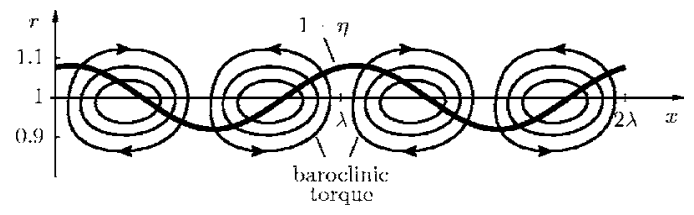

FIG. 5. Absolute mode eigenfunction of the displacement $\eta(x, r=1)$ and of the baroclinic torque $\Gamma_{b c}(x, r)$, according to Eqs. (18) and (19), for the $R / \theta=20, S=0.5$ jet.

ered as new additional variables of the generalized eigenvalue problem.

The resulting linear impulse response is included in Fig. 1 for $S=0.5$ (bullet symbols). Without the action of the baroclinic torque, all curves for $S=0.5$ and $S=1$ are found to be identical within the accuracy of the calculations, which we believe to be exact to at least four significant digits in $\omega_{0}$. It may therefore be concluded that the baroclinic torque is responsible for the onset of absolute instability in heated jets, whereas other terms involving $S$ in the continuity and energy equations are negligible. Note that the role of gravity has been neglected in these calculations, and that the baroclinic torque arises only from the base flow temperature gradient and the pressure eigenfunction.

A physical interpretation of how the baroclinic torque contributes to the destabilization of the absolute mode can be deduced from an inspection of the eigenfunction. In Fig. 5, the spatial distribution of the baroclinic torque $\Gamma_{b c}$ is superposed with the total displacement $\eta$ of the shear layer at $r=1$, both computed for the absolute mode of the $R / \theta=20, S=0.5$ jet. The displacement follows from the radial perturbation velocity according to $\partial_{t} \boldsymbol{\eta}+u_{b} \partial_{x} \eta=v^{\prime}$. For a better visualization, the spatial amplitude growth $-k_{0, i}$ has been neglected in Fig. 5. At a given time $t_{0}$, the spatial distributions are then obtained with Eq. (12) as

$$
\begin{gathered}
\eta(x, r)=\frac{F(r)}{k_{0} u_{b}-\omega_{0}} e^{i\left(k_{0, r} x-\omega_{0} t_{0}\right)}, \\
\Gamma_{b c}(x, r)=\frac{i k_{0} P(r)}{\rho_{b}^{2}} \frac{\partial \rho_{b}}{\partial r} e^{i\left(k_{0, r} x-\omega_{0} t_{0}\right)} .
\end{gathered}
$$

Both $F(r)$ and $P(r)$ have been scaled with the same arbitrary factor in Fig. 5. Equispaced isocontours of $\Gamma_{b c}(x, r)$ are shown together with the displacement of the center of the shear layer $1+\eta(r=1)$. The orientation of $\Gamma_{b c}$ is indicated by arrows. It is found that the baroclinic torque is concentrated in regions of alternating sign within the shear layer. The center of rotation of each such region, where the maximum absolute value occurs, approximately coincides with a point where the displacement is zero. The baroclinic torque arises from the shear layer undulation, and in turn it induces a further deformation that is in phase with the total shear layer displacement. Thus the temporal growth of the absolute instability mode is increased by the action of the baroclinic torque. 


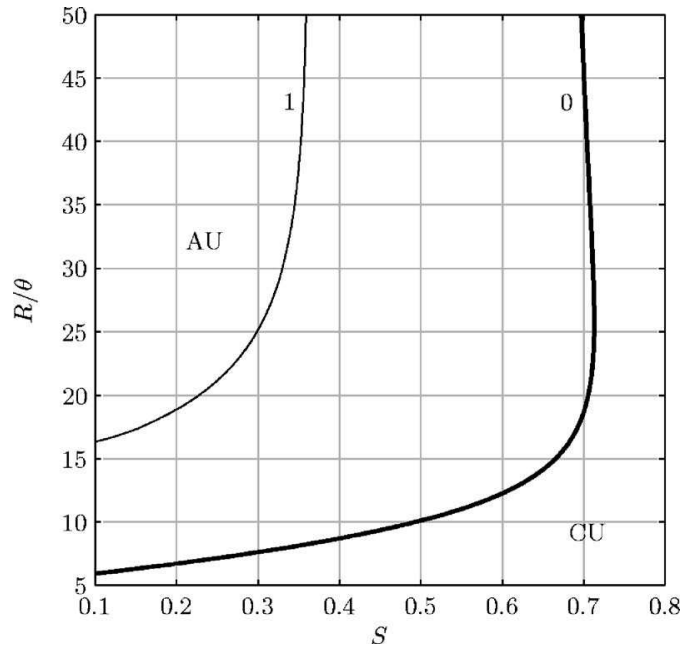

FIG. 6. Convective/absolute instability boundaries in the $S-R / \theta$ plane for $m=0$ and $m=1 . \operatorname{Re}=\infty, \mathrm{Ma}=0$.

\section{Influence of the shear layer thickness, Reynolds number, and Mach number}

The distinction between jet column and shear layer modes implies a separation of scales between the jet radius $R$ and the momentum shear layer thickness $\theta$. For low values of $R / \theta$, towards the end of the potential core in a spatially developing jet, this assumption is no longer valid. The effect of $R / \theta$ on the transition from convective to absolute instability in hot jets is explored in Fig. 6. Contours of marginal absolute instability $\left(\omega_{0, i}=0\right)$ are displayed in the $S-R / \theta$ plane for the axisymmetric and the first azimuthal mode. The absolute/ convective boundary of the axisymmetric mode is identical with Fig. 8 of Ref. 13 and also in excellent agreement with the results given in Ref. 1. Absolute instability is found to first occur for the axisymmetric jet column mode at a critical temperature ratio $S=0.713$ for $R / \theta=26$. Higher values of $R / \theta$ have a slight stabilizing effect. Below $R / \theta \sim 15$ the critical value of $S$ decreases sharply. Monkewitz and Sohn ${ }^{1}$ have shown that absolute instability of the $m=1$ mode in a top-hat jet profile requires much stronger heating than is necessary for the $m=0$ mode. However, in temporal ${ }^{24,25}$ and spatial ${ }^{7}$ jet instability studies, the $m=1$ mode has been found to display larger growth rates than its axisymmetric counterpart at very low $R / \theta$. The $m=1$ absolute instability boundary in the $S$ $-R / \theta$ plane has therefore been included in Fig. 6. It is confirmed that absolute instability always occurs first for the axisymmetric mode, even at values of $R / \theta$ as low as 6 .

Growth rates of the full linear impulse response in a thick shear layer jet with $R / \theta=5, S=1, \mathrm{Ma}=0$, and $\mathrm{Re}=\infty$ are displayed in Fig. 7 for azimuthal wave numbers $m \leq 2$. Higher-order azimuthal modes are stable everywhere. The $\sigma\left(v_{g}\right)$ distributions should be compared to the thin shear layer case $R / \theta=20$ of Fig. 4 . Note that the discontinuity that separates the axisymmetric jet column and shear layer modes in the $R / \theta=20$ jet is not observed in Fig. 7. A detailed inspection of the spatial branches reveals that the axisymmetric absolute instability mode $\left(v_{g}=0\right)$ still arises from the pinching of the $k^{+}$and $k_{1}^{-}$branches, as defined in Sec. IV A. However, at higher group velocities, both $k^{-}$branches first merge

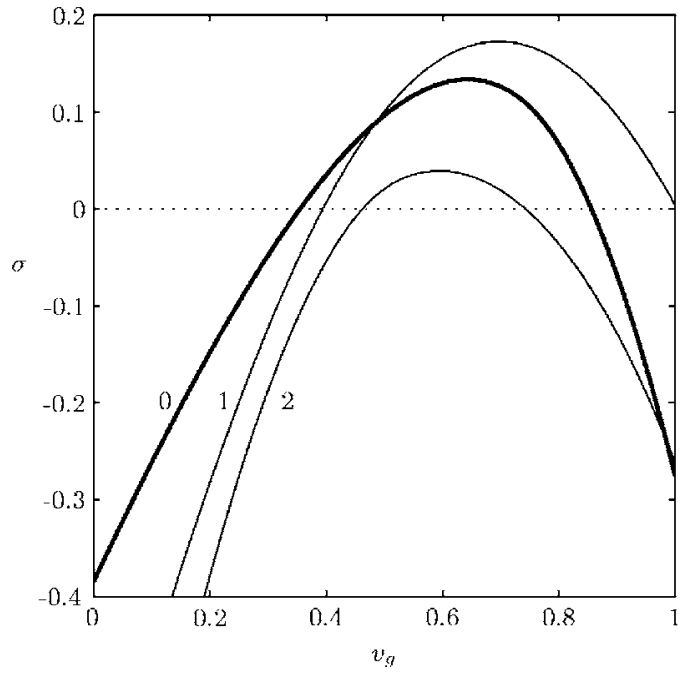

FIG. 7. Spatiotemporal growth rates in an isothermal $R / \theta=5$ thick shear layer jet for azimuthal wave numbers $m=0,1,2 . \operatorname{Re}=\infty, \mathrm{Ma}=0$.

with each other and the pinching at $\sigma\left(v_{g}\right)$ then takes place between the $k^{+}$and a combined $k_{1 / 2}^{-}$branch. This behavior is illustrated in Fig. 8 for a profile with $R / \theta=10, S=1$, and a group velocity $v_{g}=0.3$. Note that the $k_{1}^{-}$and $k_{2}^{-}$branches are no longer distinct for $\omega_{i}<0.487$, whereas pinching with the $k^{+}$branch occurs for $\omega_{i}=0.259$. The resulting spatiotemporal modes cannot be categorized as being distinctly of the jet column or shear layer type, but rather of mixed character. These mixed axisymmetric modes display lower growth rates than the formerly distinct shear layer modes. In the $R / \theta=5$ case of Fig. 7, the maximum axisymmetric temporal growth rate has now fallen below the $\sigma_{\text {max }}$ of the first helical mode. The merging of the $k_{1}^{-}$and $k_{2}^{-}$branches therefore explains the dominance of the $m=1$ over the $m=0$ mode observed in temporal stability studies of thick shear layer jets. ${ }^{24,25}$

The action of viscosity has been neglected in all instability calculations presented so far. If the Reynolds number

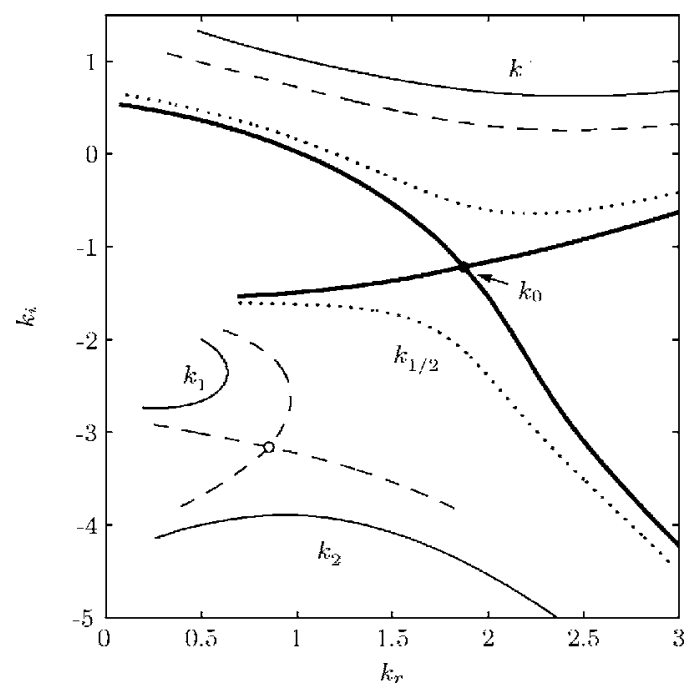

FIG. 8. Branches in the complex $k$ plane for $v_{g}=0.3, R / \theta=10, S=1$, Re $=\infty$, and $\mathrm{Ma}=0$. Four constant values of $\omega_{i}: 0.6$ (thin solid), 0.487 (dashed), 0.3 (dotted), 0.259 (thick solid). 


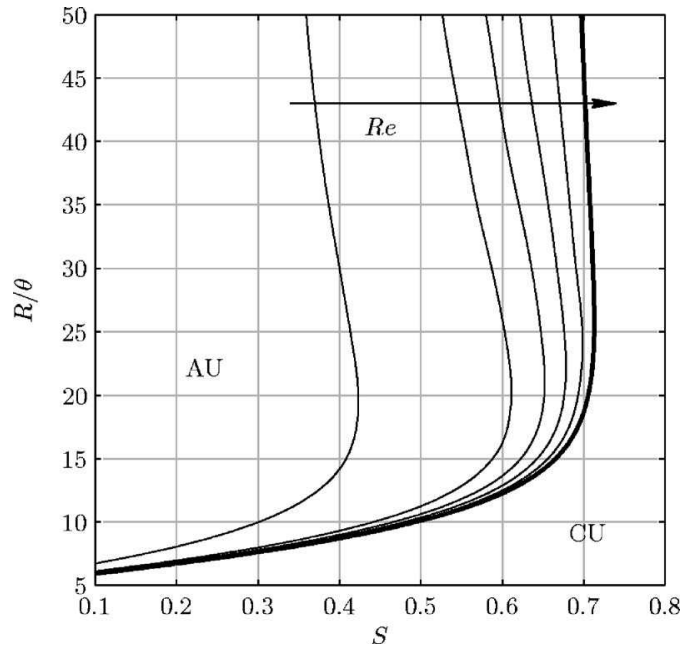

FIG. 9. Convective/absolute instability boundaries in the $S-R / \theta$ plane for $\mathrm{Ma}=0 . \mathrm{Re}=100,500,1000,2000,5000$ (thin lines); $\mathrm{Re}=\infty$ (thick line).

takes on finite values, the inviscid instability modes described above are affected by viscous damping. The effect of viscosity on the absolute instability of the axisymmetric mode is exhibited in Fig. 9. As the Reynolds number decreases, the absolute/convective transition is delayed towards lower values of $S$. At high Reynolds numbers, viscosity first affects the critical temperature ratio at high values of $R / \theta$. The slight stabilizing effect of $R / \theta \rightarrow \infty$ observed in the inviscid limit becomes more pronounced in viscous jets.

Corresponding curves of the absolute instability boundary for various Mach numbers at $\mathrm{Re}=\infty$ are presented in Fig. 10. In agreement with earlier studies, ${ }^{1,13}$ the stabilization of the jet column mode is quite significant already at moderate Mach numbers. The offset $\Delta S$ of the convective/absolute transition that is induced by a given Mach number over the interval $0 \leq \mathrm{Ma} \leq 0.5$ is found to be uniform for all $R / \theta$, and can be well approximated as $\Delta S=-1.4 \mathrm{Ma}^{2}$.

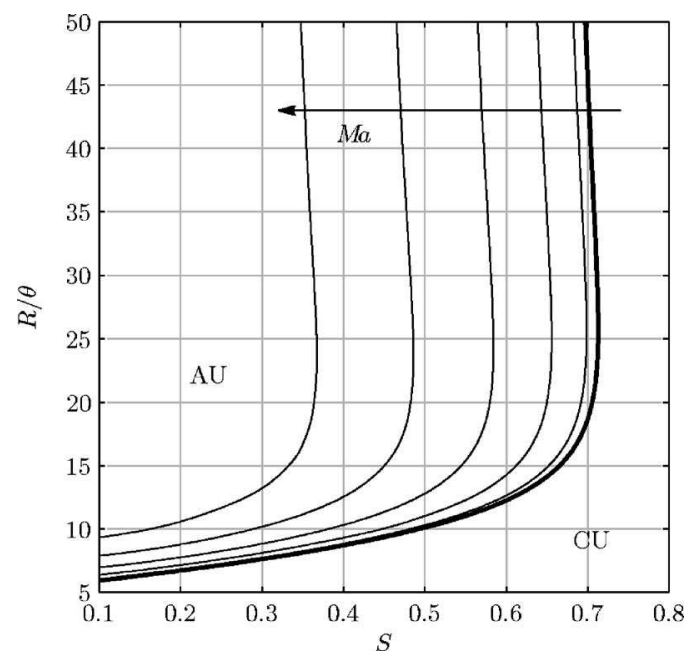

FIG. 10. Convective/absolute instability boundaries in the $S-R / \theta$ plane for $\mathrm{Re}=\infty . \mathrm{Ma}=0$ (thick line), $\mathrm{Ma}=0.1,0.2,0.3,0.4,0.5$ (thin lines).

\section{CONCLUSIONS}

The linear impulse response of isothermal and heated round jets has been investigated for axisymmetric and higher-order azimuthal modes. A fully compressible formulation of the spatial instability problem has been developed, and results for the linear impulse response have been presented in terms of complex frequency and wave number as functions of the group velocity. In agreement with Jendoubi and Strykowski, ${ }^{13}$ the absolute mode $\left(v_{g}=0\right)$ in jets without counterflow has been found to always be of the axisymmetric jet column type. However, shear layer modes have been shown to dominate the linear impulse response for high group velocities in thin shear layer jets. Axisymmetric and helical modes are in close competition throughout this portion of the wave packet. The most amplified spatiotemporal mode in thin shear layer jets is of the shear layer type. Jetcolumn-type solutions are only admitted for axisymmetric perturbations, and their prevalence over shear layer modes is restricted to a small range of low group velocities.

In the presence of sufficiently strong heating, the jet column mode becomes absolutely unstable. In excellent agreement with Refs. 1 and 13, the critical temperature ratio for this transition has been determined as $S_{c}=0.713$ for a shear layer thickness given by $R / \theta=26$. The onset of absolute instability in heated jets has been demonstrated to arise from the action of the baroclinic torque, and a physical interpretation has been proposed. An inspection of the absolute mode eigenfunction has shown that the additional deformation induced by the baroclinic torque is in phase with the total shear layer deformation. If the baroclinic torque is eliminated from the dispersion relation, the linear impulse responses of heated and isothermal jets in the inviscid, zero-Mach-number limit are identical.

The clearcut duality of jet column versus shear layer modes is lost as the shear layer thickness approaches the jet radius. The axisymmetric linear impulse response of an $R / \theta=5$ jet profile displays a smooth transition between the formerly clearly divided jet column/shear layer dominated regions of the wave packet. It has been found from examination of the complex $k$ branches that in thick shear layer jets, modes of a mixed character arise from the merging of jet column and shear layer type $k^{-}$branches prior to the pinching with the $k^{+}$branch. This mixed character accounts for a lowered maximum temporal growth rate of axisymmetric disturbances relative to their helical counterparts, as it has been observed in temporal instability studies of thick shear layer jets. ${ }^{24,25}$

The influence of viscosity on the absolute/convective transition has been analyzed by tracking the critical temperature ratio as a function of $R / \theta$ for Reynolds numbers between 100 and infinity. As may have been expected, viscosity has a purely stabilizing effect, but its influence lessens for low values of $R / \theta$. In contrast, a finite Mach number delays the critical temperature ratio by a constant offset for all $R / \theta$. 


\section{ACKNOWLEDGMENTS}

The authors are grateful to C. Olendraru and A. Sellier for their help in the development of the instability code. Fruitful comments from C. Cossu and J.-M. Chomaz have also contributed to the present study. The friendly Ladhyx work environment is warmly appreciated.

\section{APPENDIX: THE COMPRESSIBLE SPATIAL EIGENVALUE PROBLEM}

If the equations of motion (6)-(8) are linearized about the base flow defined in Sec. II, and all perturbation quantities are expressed in normal mode form (12), the following system of equations is obtained:

Continuity:

$$
r \omega D+\left(\rho_{b}+r \rho_{b}^{\prime}\right) F+r \rho_{b} F^{\prime}+m \rho_{b} G=-r u_{b} k D-r \rho_{b} k H .
$$

$x$ momentum:

$$
\left(i r^{2} \rho_{b} \omega-\frac{m^{2}}{\operatorname{Re}}\right) H+\frac{r}{\operatorname{Re}} H^{\prime}+\frac{r^{2}}{\operatorname{Re}} H^{\prime \prime}-i r^{2} \rho_{b} u_{b}^{\prime} F=i r^{2} \rho_{b} u_{b} k H+\frac{r}{3 \operatorname{Re}} k F+\frac{r^{2}}{3 \operatorname{Re}} k F^{\prime}+\frac{m r}{3 \operatorname{Re}} k G+i r^{2} k P+\frac{4 r^{2}}{3 \mathrm{Re}} k^{2} H .
$$

$r$ momentum:

$$
\left(i r^{2} \rho_{b} \omega-\frac{4+3 m^{2}}{3 \operatorname{Re}}\right) F+\frac{4 r}{3 \operatorname{Re}} F^{\prime}+\frac{4 r^{2}}{3 \operatorname{Re}} F^{\prime \prime}-\frac{7 m}{3 \operatorname{Re}} G+\frac{m r}{3 \operatorname{Re}} G^{\prime}+i r^{2} P^{\prime}=-\frac{r^{2}}{3 \operatorname{Re}} k H^{\prime}+i r^{2} \rho_{b} u_{b} k F+\frac{r^{2}}{\operatorname{Re}} k^{2} F .
$$

$\phi$ momentum:

$$
\frac{7 m}{3 \operatorname{Re}} F+\frac{m r}{3 \operatorname{Re}} F^{\prime}+\left(-i r^{2} \rho_{b} \omega+\frac{3+4 m^{2}}{3 \operatorname{Re}}\right) G-\frac{r}{\operatorname{Re}} G^{\prime}-\frac{r^{2}}{\operatorname{Re}} G^{\prime \prime}+i m r P=-\frac{m r}{3 \operatorname{Re}} k H-i r^{2} \rho_{b} u_{b} k G-\frac{r^{2}}{\operatorname{Re}} k^{2} G
$$

Energy:

$$
\begin{aligned}
\frac{1}{\gamma-1} & {\left[\frac{i \omega r^{2}}{\rho_{b}}-\frac{\gamma}{\rho_{b}^{2} \operatorname{Re} \operatorname{Pr}}\left(m^{2}-6 r^{2} \frac{\rho_{b}^{\prime 2}}{\rho_{b}^{2}}+2 r \frac{\rho_{b}^{\prime}}{\rho_{b}}+2 r^{2} \frac{\rho_{b}^{\prime \prime}}{\rho_{b}}\right)\right] D+\frac{\gamma^{2} \mathrm{Ma}^{2} r^{2}}{(\gamma-1) \rho_{b}^{2} \operatorname{Re} \operatorname{Pr}}\left(1-4 r \frac{\rho_{b}^{\prime}}{\rho_{b}}\right) D^{\prime}+\frac{\gamma r^{2}}{(\gamma-1) \rho_{b}^{2} \operatorname{Re} \operatorname{Pr} D^{\prime \prime}} } \\
& -\frac{2 \gamma \mathrm{Ma}^{2}}{\operatorname{Re}} r^{2} u_{b}^{\prime} H^{\prime}+\left[i r-\frac{i r^{2} \rho_{b}^{\prime}}{(\gamma-1) \rho_{b}}\right] F+i r^{2} F^{\prime}+i m r G+\frac{\gamma \mathrm{Ma}^{2}}{\gamma-1}\left[-i r^{2} \omega-\frac{\gamma}{\rho_{b} \operatorname{Re} \operatorname{Pr}}\left(2 \frac{r^{2} \rho_{b}^{\prime 2}}{\rho_{b}^{2}}-\frac{r^{2} \rho_{b}^{\prime \prime}}{\rho_{b}}-\frac{r \rho_{b}^{\prime}}{\rho_{b}}-m^{2}\right)\right] P \\
& +\frac{\gamma^{2} \mathrm{Ma}^{2} r}{(\gamma-1) \rho_{b} \operatorname{Re} \operatorname{Pr}}\left(2 r \frac{\rho_{b}^{\prime}}{\rho_{b}}-1\right) P^{\prime}-\frac{\gamma^{2} \mathrm{Ma}^{2} r^{2}}{(\gamma-1) \rho_{b} \operatorname{Re} \operatorname{Pr}} P^{\prime \prime}=i \frac{r^{2} u_{b}}{(\gamma-1) \rho_{b}} k D-i r^{2} k H-\frac{2}{\operatorname{Re}^{2}} \gamma \mathrm{Ma}^{2} r^{2} u_{b}^{\prime} k F-i \frac{\gamma \mathrm{Ma}^{2}}{\gamma-1} r^{2} u_{b} k P \\
& +\frac{\gamma r^{2}}{(\gamma-1) \rho_{b}^{2} \operatorname{Re} \operatorname{Pr}} k^{2} D-\frac{\gamma^{2} \mathrm{Ma}^{2} r^{2}}{(\gamma-1) \rho_{b} \operatorname{Re} \operatorname{Pr}} k^{2} P .
\end{aligned}
$$

Primes in the above equations denote radial derivatives. In the incompressible limit $\mathrm{Ma}=0, \rho_{b}^{\prime} \equiv 0, D \equiv 0$, the continuity and energy Eqs. (A1) and (A5) are identical, and Eqs. (A1)-(A4) are equivalent to the incompressible formulation given by Ash and Khorrami. ${ }^{18}$

The system (A1)-(A5) may now be written in the form of a generalized eigenvalue problem

$$
\mathbf{A X}=k \mathbf{B X}
$$




\section{Boundary conditions}

In the coordinate singularity at $r=0$, compatibility conditions ${ }^{24}$ must be imposed so as to ensure bounded solutions for all perturbations. Khorrami et al. ${ }^{15}$ obtained these conditions for $H, F, G, P$ in a formal way by requiring the azimuthal derivatives of velocity and pressure perturbations to vanish as $r \rightarrow 0$. Accordingly, density variations $\rho^{\prime}$ must obey

$$
\lim _{r \rightarrow 0} \frac{\partial \rho^{\prime}}{\partial \phi}=i m D(0)=0 .
$$

Together, these requirements impose (see Ref. 18)

$$
\begin{aligned}
& \left.\begin{array}{l}
F(0)=G(0)=0 \\
D(0), H(0) \text { and } P(0) \text { finite }
\end{array}\right\} \text { for } m=0, \\
& \left.\begin{array}{l}
\begin{array}{l}
F(0) \pm G(0)=0, F^{\prime}(0)=0 \\
D(0)=H(0)=P(0)=0
\end{array} \\
\begin{array}{l}
D(0)=H(0)=F(0) \\
=G(0)=P(0)=0
\end{array}
\end{array}\right\} \text { for } m= \pm 1,
\end{aligned}
$$

Explicit expressions for $D(0), H(0)$, and $P(0)$ in the $m=0$ case are further deduced from Taylor expansions of Eqs. (A2), (A3), and (A5) around the jet centerline. In the limit $r \rightarrow 0$, these equations admit

$$
\begin{aligned}
& H^{\prime}(0)=0, \\
& P^{\prime}(0)=i \frac{2}{\operatorname{Re}} F^{\prime \prime}(0), \\
& D^{\prime}(0)=\gamma \operatorname{Ma}^{2} P^{\prime}(0) .
\end{aligned}
$$

According to Ash and Khorrami, ${ }^{18}$ all eigenfunctions decay exponentially as $r \rightarrow \infty$. The far-field conditions to the spatial eigenvalue problem for all $m$ are simply

$$
D(\infty)=H(\infty)=F(\infty)=G(\infty)=P(\infty)=0 .
$$

\section{Chebyshev collocation}

Following Ref. 15, the eigenfunctions $(D, H, F, G, P)$ are mapped from the physical domain $0 \leq r \leq r_{\max }$ onto the Chebyshev interval $-1 \leq \xi \leq 1$, where they are discretized in $N$ collocation points,

$$
\xi_{j}=\cos \left(\frac{j \pi}{N-1}\right), \quad j=0, \ldots, N-1 .
$$

For the problem at hand, a suitable mapping function $\xi(r)$ had to be conceived to concentrate most collocation points within the shear layer region of the physical domain. With the two-parameter transformation

$$
\xi(r)=\frac{r_{c}}{2 r}-\sqrt{1+\frac{r_{c}^{2}}{4 r^{2}}+\frac{2 r_{c}}{r_{\text {max }}}-\frac{r_{c}}{r}},
$$

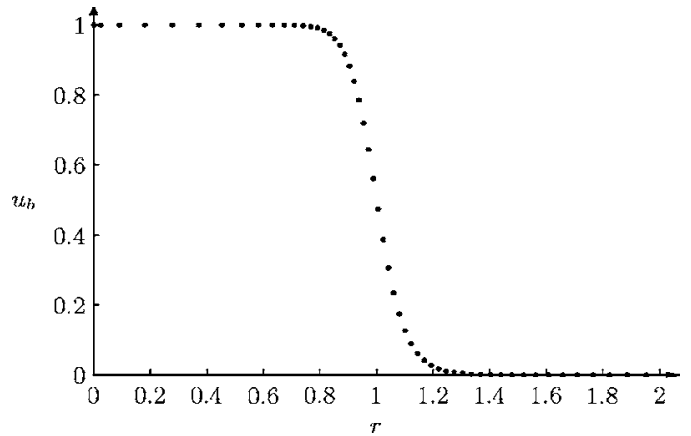

FIG. 11. Discrete resolution of a $R / \theta=20$ velocity profile as obtained from transformation (A13) with $r_{c}=1.8, r_{\max }=100, N=100$.

$$
r(\xi)=r_{c} \frac{1-\xi}{1-\xi^{2}+2 r_{c} / r_{\max }},
$$

approximately half of the points $r_{j}=r\left(\xi_{j}\right)$ are placed in the interval $0 \leq r \leq r_{c}$, concentrated around $r=r_{c} / 2$. The far-field conditions (A11) are imposed at $r_{\max } \gg 1$. Values of $r_{c}=1.8$ and $r_{\max }=100$ have been used in all calculations. The discretization of a $R / \theta=20$ velocity profile obtained with these settings and with a typical resolution $N=100$ is shown in Fig. 11.

${ }^{1}$ P. A. Monkewitz and K. Sohn, "Absolute instability in hot jets," AIAA J. 26, 911 (1988).

${ }^{2}$ P. A. Monkewitz, D. W. Bechert, B. Barsikow, and B. Lehmann, "Selfexcited oscillations and mixing in a heated round jet," J. Fluid Mech. 213, 611 (1990)

${ }^{3}$ K. R. Sreenivasan, S. Raghu, and D. Kyle, "Absolute instability in variable density round jets," Exp. Fluids 7, 309 (1989).

${ }^{4}$ S. Boujemaa, M. Amielh, and M. P. Chauve, "Analyse spatio-temporelle de jets axisymétriques d'air et d'hélium," C. R. Mec. 332, 933 (2004).

${ }^{5}$ B. S. Yildirim and A. K. Agrawal, "Full-field measurements of selfexcited oscillations in momentum-dominated helium jets," Exp. Fluids 38, 161 (2005).

${ }^{6}$ A. Michalke, "Instabilität eines kompressiblen runden Freistrahls unter Berücksichtigung des Einflusses der Strahlgrenzschichtdicke," Z. Flugwiss. 19, 319 (1971) [NASA Tech. Memo. Report No. 75190 (1977) (English translation of the original German article)].

${ }^{7}$ A. Michalke, "Survey on jet instability theory," Prog. Aerosp. Sci. 21, 159 (1984).

${ }^{8}$ W. Blumen, P. G. Drazin, and D. F. Billings, "Shear layer instability of an inviscid compressible fluid. Part 2," J. Fluid Mech. 71, 305 (1975).

${ }^{9}$ R. Briggs, Electron-stream interaction with plasmas, Research Monograph series, Vol. 29 (MIT Press, Cambridge, MA, 1964).

${ }^{10}$ A. Bers, in Handbook of Plasma Physics 1, edited by M. N. Rosenbluth and R. Z. Sagdeev (North-Holland, Amsterdam, 1983).

${ }^{11}$ P. Huerre and P. A. Monkewitz, "Absolute and convective instabilities in free shear layers," J. Fluid Mech. 159, 151 (1985).

${ }^{12}$ S. Pavithran and L. G. Redekopp, "The absolute-convective transition in subsonic mixing layers," Phys. Fluids A 1, 1736 (1989).

${ }^{13}$ S. Jendoubi and P. J. Strykowski, "Absolute and convective instability of axisymmetric jets with external flow," Phys. Fluids 6, 3000 (1994).

${ }^{14}$ P. Huerre, "Open shear flow instabilities," in Perspectives in Fluid Dynamics, edited by G. K. Batchelor, H. K. Moffatt, and M. G. Worster (Cambridge University Press, Cambridge, 2000), pp. 159-229.

${ }^{15}$ M. R. Khorrami, M. R. Malik, and R. L. Ash, "Applications of spectral collocation techniques to the stability of swirling flows," J. Comput. Phys. 81, 206 (1989).

${ }^{16} \mathrm{P}$. Huerre and M. Rossi, "Hydrodynamic instabilities in open flows," in Hydrodynamics and Nonlinear Instabilities, edited by C. Godreche and P. Manneville (Cambridge University Press, Cambridge, 1998), pp. 81-294.

${ }^{17}$ I. Delbende, J.-M. Chomaz, and P. Huerre, "Absolute/convective instabilities in the Batchelor vortex: a numerical study of the linear impulse response," J. Fluid Mech. 355, 229 (1998). 
${ }^{18}$ R. L. Ash and M. R. Khorrami, "Vortex stability," in Fluid Vortices, edited by S. I. Green (Kluwer, Dordrecht, 1995), pp. 317-372.

${ }^{19}$ C. Olendraru and A. Sellier, "Viscous effects in the absolute-convective instability of the Batchelor vortex," J. Fluid Mech. 459, 371 (2002).

${ }^{20}$ L. Lesshafft, P. Huerre, P. Sagaut, and M. Terracol, "Nonlinear global modes in hot jets," J. Fluid Mech. 554, 393 (2006).

${ }^{21}$ P. Huerre and P. A. Monkewitz, "Local and global instabilities in spatially developing flows," Annu. Rev. Fluid Mech. 22, 473 (1990).

${ }^{22}$ T. Loiseleux, J.-M. Chomaz, and P. Huerre, "The effect of swirl on jets and wakes: Linear instability of the Rankine vortex with axial flow," Phys. Fluids 10, 1120 (1998).

${ }^{23}$ M. C. Soteriou and A. F. Ghoniem, "Effects of the free-stream density ratio on free and forced spatially developing shear layers," Phys. Fluids 7, 2036 (1995).

${ }^{24}$ G. K. Batchelor and A. E. Gill, "Analysis of the stability of axisymmetric jets," J. Fluid Mech. 14, 529 (1962).

${ }^{25}$ F. Gallaire and J.-M. Chomaz, "Mode selection in swirling jet experiments: a linear stability analysis,” J. Fluid Mech. 494, 223 (2003). 